\title{
Perfil acadêmico, crenças e autoeficácia em pesquisa de enfermeiros clínicos: implicações para o Programa de Pesquisa de Enfermagem de um Hospital na Jornada Magnet ${ }^{\circledR}$
}

\author{
Academic profile, beliefs, and self-efficacy in research of clinical nurses: \\ implications for the Nursing Research Program in a Magnet Journey ${ }^{\text {TM }}$ hospital \\ Eliseth Ribeiro Leão ${ }^{1}$, Olga Guilhermina Farah ${ }^{1}$, Elisa Aparecida Alves Reis ${ }^{1}$, \\ Claudia Garcia de Barros ${ }^{1}$, Cristina Satoko Mizoi ${ }^{1}$
}

\section{RESUMO}

Objetivo: Descrever o perfil acadêmico, a experiência em pesquisa, as crenças e a autoeficácia em pesquisa dos enfermeiros clínicos de um hospital em Jornada Magnet ${ }^{\circledR}$. Métodos: Estudo descritivo, tipo survey, para avaliar perfil acadêmico, experiência em pesquisa, crenças e habilidades de pesquisa dos enfermeiros clínicos. A análise foi dividida em características demográficas; perfil acadêmico/científico (titulação acadêmica, participação em grupos de pesquisa, envolvimento em publicações, atividades de ensino, conferências científicas e apresentação de posteres); crenças relacionadas à Pesquisa em Enfermagem (habilidades, benefícios para a carreira, reputação da instituição, para 0 cuidado do paciente, satisfação no trabalho); e autoeficácia em pesquisa (conduzir revisão de literatura, avaliar a qualidade dos estudos, usar teoria; compreender as evidências e escrita científica: facilidade para colocar as ideias no papel, reconhecer e adaptar o texto para o leitor, escrever obedecendo os padrões requeridos pela ciência, escrever com objetividade, clareza e precisão; inserir as referências apropriadamente, usar corretamente a ortografia e gramática; escrever textos em inglês). Resultados: A maioria dos enfermeiros clínicos tinha pouca experiência em pesquisa, todavia, demonstraram crenças positivas e percepção de habilidades de pesquisa bem desenvolvidas. Conclusão: Nossos achados devem contribuir para a elaboração de programas de pesquisa que objetivem facilitar o engajamento dos enfermeiros clínicos no desenvolvimento de projetos científicos.

Descritores: Pesquisa em enfermagem clínica; Pesquisa em enfermagem; Pesquisa em avaliação de enfermagem

\section{ABSTRACT}

Objective: To describe the academic profile, research experience, beliefs, and self-efficacy in research of clinical nurses in a Magnet
Journey $^{\mathrm{TM}}$ hospital. Methods: Quantitative descriptive designed to assess research experience of clinical nurses. The survey was divided into demographics characteristics; scientific/academic profile (Nursing degree; membership in academic research groups, involvement in papers, teaching activities, scientific conferences, and posters presented); beliefs related to nursing research (about skills, benefits to career, reputation of institution, patient care; job satisfaction level); and Research Self-Efficacy (conducting literature review; evaluating quality of studies; using theory; understanding evidence; and scientific writing: putting ideas on paper easily; recognize and adapt the text to the reader; write to the standards required by science; write with objectivity, logical sequence, coherence, simplicity, clarity, and precision; insert the references in the text correctly; write the references appropriately; use correct spelling and grammar; write texts in English). Results: Most clinical nurses had low research experience, yet had positive beliefs in and perception of well-developed research skills. Conclusion: Our findings should contribute to the preparation of research programs aimed at facilitating the engagement of clinical nurses in the development of scientific projects.

Keywords: Clinical nursing research; Nursing research; Nursing evaluation research

\section{INTRODUÇÃO}

Os cuidados de enfermagem caracterizam-se por competência técnica e humanística, bem como conhecimento científico nesse campo. A alta qualidade do atendimento depende de todas essas dimensões.

Uma maneira bem estabelecida de promover tais habilidades é por meio de apoio e motivação da pes-

\footnotetext{
Trabalho realizado no Hospital Israelita Albert Einstein, São Paulo, SP, Brasil.

1 Hospital Israelita Albert Einstein, São Paulo, SP, Brasil.

Autor correspondente: Eliseth Ribeiro Leão - Avenida Albert Einstein, 627/701 - Morumbi - CEP: 05652-900 - São Paulo, SP, Brasil - Tel: (11) 2151-1032 - E-mail: eliseth.leao@einstein.br

Data de submissão: 24/7/2013 - Data de aceite: 9/11/2013
}

Conflitos de interesse: não há. 
quisa em enfermagem. $\mathrm{O}$ contato com informações cientificamente estruturadas permite que a equipe de enfermagem fique atualizada nesse campo de forma constante, que avalie as suas práticas e ajude a crescer em termos profissionais, incorporando novas maneiras de pensar e cuidar de seus pacientes ${ }^{(1)}$.

Vários fatores contribuíram para a implantação de programas de pesquisa em enfermagem em instituições privadas, e muitos estão relacionados à necessidade de estabelecer padrões de qualidade internacional nesses centros. Um caso de apoio crescente ao desenvolvimento da pesquisa em enfermagem refere-se aos requisitos associados à designação do Magnet ${ }^{\circledR}$. O Programa de Reconhecimento Magnet ${ }^{\circledR}$ foi desenvolvido pelo American Nurses Credentialing Center (ANCC) para reconhecer as organizações de assistência à saúde que possuem excelência em serviços de enfermagem ${ }^{(2)}$. Em hospitais com designação Magnet ${ }^{\circledR}$ e hospitais da Jornada Magnet $^{\circledR}$, as unidades de pesquisa são criadas para facilitar o desenvolvimento da pesquisa. Mas esse é apenas um primeiro passo no processo. É sempre preciso ter o apoio de um enfermeiro-pesquisador (cientistas de enfermagem com doutorado) ${ }^{(3)}$.

Desenvolver uma cultura de pesquisa fora do mundo acadêmico não é uma tarefa fácil, especialmente para profissionais com dedicação total aos cuidados de enfermagem. A pesquisa em enfermagem é, em geral, ainda muito restrita às universidades, e os enfermeiros clínicos têm muitas dificuldades para desenvolver projetos de pesquisa. Em primeiro lugar, como implantar programas de pesquisa que promovam o engajamento de enfermeiros clínicos na pesquisa em enfermagem? Para tanto, é necessário conhecer o passado acadêmico dos enfermeiros, assim como suas crenças e autoeficácia em pesquisa, para adotar as estratégias mais apropriadas. Além disso, ainda não há um consenso sobre o melhor modelo de treinamento e apoio à pesquisa, ou como engajar de maneira eficaz os enfermeiros clínicos na pesquisa. Desse modo, é necessário um programa abrangente e multifacetado para preencher essa lacuna entre pesquisa e prática ${ }^{(4)}$. Isso é possível somente por meio de um diagnóstico detalhado do passado, experiências e crenças dos enfermeiros clínicos em relação à pesquisa.

Neste estudo, relatamos os resultados de uma avaliação detalhada do perfil acadêmico, experiência em pesquisa, crenças e autoeficácia em pesquisa de enfermeiros clínicos funcionários do Hospital Israelita Albert Einstein (HIAE), em São Paulo, Brasil, que é a primeira instituição na América Latina a se candidatar à designação Magnet ${ }^{\circledR}$. A compreensão do perfil e das idiossincrasias dessa população de enfermeiros capacitou a preparação de um programa de pesquisa, com o objetivo de facilitar o engajamento de enfermeiros clínicos no desenvolvimento de projetos científicos. O conhecimento dos fatores associados à implantação bem-sucedida das práticas de pesquisa em centros não acadêmicos, particularmente naqueles que buscam a designação Magnet ${ }^{\circledR}$, pode inspirar outras organizações em outros contextos a iniciar ou consolidar essa atividade nos cuidados à beira do leito.

\section{OBJETIVO}

Diagnosticar o perfil acadêmico, a experiência em pesquisa, as crenças e a autoeficácia na pesquisa de enfermeiros clínicos em hospital na Jornada Magnet ${ }^{\circledR}$.

\section{MÉTODOS}

Foi realizada uma pesquisa eletrônica descritiva utilizando-se uma amostra de conveniência de 165 enfermeiros clínicos $(20 \%$ do total de enfermeiros clínicos até janeiro de 2012) do HIAE, hospital privado, localizado em São Paulo e primeira instituição da América Latina aceito na Jornada Magnet ${ }^{\circledR}$ em outubro de 2011. O convite para participar e responder aos questionários foram enviados por e-mail. A participação foi voluntária, e os dados foram processados em setembro de 2012. Todos os participantes assinaram o Formulário de Consentimento Informado para este estudo.

A pesquisa foi dividida em quatro subseções: (1) Dados Demográficos (7 perguntas); (2) Perfil Acadêmico/ Científico (18 perguntas); (3) Crenças Relacionadas à Pesquisa em Enfermagem (8 perguntas; Escala Likert), com base nas declarações propostas por Apleton et al. ${ }^{(5)}$; e (4) Autoeficácia em Pesquisa (22 itens, escala de 5 pontos) proposta por Sweson-Britt e Reineck ${ }^{(6)}$. As atividades listadas na Escala de Autoeficácia em Pesquisa de Enfermagem foram traduzidas para o português e foram adicionados um item relacionado à pesquisa literária em bases de dados na América Latina e um item relacionado à redação em inglês (oito itens: colocar as ideias facilmente no papel; reconhecer e adaptar o texto para o leitor; redigir com os padrões exigidos pela ciência; redigir com objetividade, sequência lógica, coerência, simplicidade, clareza e precisão; inserindo corretamente as referências no texto; redigir corretamente as referências; usar ortografia e gramática corretas; redigir textos em inglês). Esse instrumento não classifica pontos, não requerendo validação, pois serve apenas como guia para a simples quantificação das habilidades.

As variáveis categóricas foram descritas como frequências absolutas e porcentagens. As variáveis qualitativas foram expressas como variações medianas. As comparações 
entre as medidas de crenças e habilidades em diferentes categorias de variáveis de perfis foram realizadas com a utilização dos testes Mann-Whitney e Kruskal-Wallis.

Para as pontuações de crenças e habilidades, foram adicionadas as respostas aos itens, e os valores das pontuações foram os seguintes: pontuação mínima $=1 \mathrm{x}$ número de itens $=$ acordo ou crença máxima; pontuação máxima $=5 \mathrm{x}$ número de itens $=$ acordo ou crença mínima. Assim, as pontuações padronizadas mínimas e máximas foram zero e 10 (independentemente do número de itens adicionados), representando, respectivamente, os níveis máximos e mínimos de acordo ou crença. Os testes estatísticos foram realizados com Statistical Package for the Social Sciences (SPSS), versão 17. A significância foi considerada como $\mathrm{p} \leq 0.05$.

O projeto foi aprovado pelo Comitê de Ética em Pesquisa, sob número 36.268 .

\section{RESULTADOS}

\section{Perfil acadêmico dos participantes}

Dos 165 participantes da pesquisa, a maioria $(87,3 \%)$ era formada por enfermeiros clínicos, seguida por gestores de enfermagem $(6,1 \%)$, administradores de enfermagem $(5,5 \%)$, e enfermeiros pesquisadores $(1,2 \%)$. Embora todos os participantes fossem graduados em enfermagem, a maioria $(72,7 \%)$ tinha concluído a graduação há 5 anos ou mais antes da entrevista. A maioria dos participantes $(85,5 \%)$ também tinha um título de especialização, sendo um total de $12,7 \%$ com título de mestrado e $3 \%$, de doutorado.

A análise da experiência em pesquisa revelou que somente $13,3 \%$ participavam de grupos de pesquisa acadêmica. Ainda, a maioria dos participantes relatou uma experiência razoável e boa $(40 \%$ e $17,6 \%$, respectivamente), com apenas $8,5 \%$ e $33,9 \%$ tendo relatado nenhuma ou muito pouca experiência em pesquisa. De maneira semelhante, a análise mostrou que apenas
17,6\% apresentaram resultados de pesquisa em congressos científicos e $9,7 \%$ tinham publicado um manuscrito nos 2 anos antes da entrevista. A maioria (77,5\%) dos respondentes esperava participar de atividades de pesquisa. A participação nas atividades de docência foi relatada por cerca de um terço $(34,5 \%)$ dos respondentes.

\section{Crenças dos enfermeiros clínicos e 0 impacto na pes- quisa em enfermagem}

A tabela 1 mostra que a maioria dos enfermeiros clínicos tinha crenças positivas sobre os benefícios da pesquisa em enfermagem, predominantemente sobre o impacto da pesquisa na imagem da empresa, no desenvolvimento do trabalho em equipe e em assistência aos pacientes.

\section{Autoeficácia em pesquisa de enfermagem}

A figura 1 mostra pontuações mais altas nas habilidades relacionadas ao uso do conhecimento teórico, compreensão de evidências e capacidade de conduzir revisões literárias. A capacidade de analisar criticamente artigos científicos em outros idiomas foi pouco avaliada.

A análise das habilidades em pesquisa global dessa população indicou que tais habilidades dependiam do grau de complexidade de cada atividade de pesquisa. As taxas de concordância (respostas 'concordo' e 'concordo totalmente') relacionadas com a capacidade de revisar a literatura variaram entre 51,2 e 87,3\% (média de 68,7\%). As pontuações atribuídas às habilidades de análise crítica variaram entre 38,1 e 60,6\% (média 49,5\%) e aquelas atribuídas à prática baseada em evidência e em conhecimentos teóricos variaram entre 75,1 e $89,7 \%$ (média $82,2 \%$ ). As habilidades de redação científica tiveram pontuações que variaram entre 67,3 e 84,2\% (média de $73,6 \%$ ). A maioria dos respondentes acreditava que era capaz de ler e compreender um artigo científico redigido em inglês ou espanhol (70,9 e 69,1\%, respectivamente).

Tabela 1. Crenças relacionadas ao impacto da pesquisa em enfermagem na qualidade dos cuidados ao paciente e na vida profissional

\begin{tabular}{|c|c|c|c|c|c|c|}
\hline Crenças & $\begin{array}{c}\text { Acredito } \\
\text { totalmente } \\
\text { n }(\%)\end{array}$ & $\begin{array}{l}\text { Acredito } \\
\text { n (\%) }\end{array}$ & $\begin{array}{c}\text { Não acredito nem } \\
\text { desacredito } \\
\text { n (\%) }\end{array}$ & $\begin{array}{l}\text { Não acredito } \\
\text { n }(\%)\end{array}$ & $\begin{array}{l}\text { Não acredito } \\
\text { totalmente } \\
n(\%)\end{array}$ & $\begin{array}{l}\text { Total } \\
\text { n (\%) }\end{array}$ \\
\hline Garante que os padrões de cuidados aos pacientes sejam aprimorados & $84(50,9)$ & $75(45,5)$ & $6(3,6)$ & $0(0)$ & $0(0)$ & $165(100)$ \\
\hline Beneficia a equipe em termos de desenvolver suas habilidades & $83(50,3)$ & $78(47,3)$ & $4(2,4)$ & $0(0)$ & $0(0)$ & $165(100)$ \\
\hline Ajuda a melhorar a maneira como a equipe trabalha junto & $76(46,1)$ & $83(50,3)$ & $6(3,6)$ & $0(0)$ & $0(0)$ & $165(100)$ \\
\hline Eleva o perfil e a reputação da unidade & $89(53,9)$ & $68(41,2)$ & $6(3,6)$ & $2(1,2)$ & $0(0)$ & $165(100)$ \\
\hline Eleva o perfil e a reputação do centro & $97(58,8)$ & $65(39,4)$ & $3(1,8)$ & $0(0)$ & $0(0)$ & $165(100)$ \\
\hline Ajuda a aprimorar meu desenvolvimento profissional/carreira & $103(62,4)$ & $56(33,9)$ & $5(3,0)$ & $1(0,6)$ & $0(0)$ & $165(100)$ \\
\hline $\begin{array}{l}\text { Promove o envolvimento do paciente e dos cuidados prestados de } \\
\text { mostrando como o departamento é organizado e como funciona }\end{array}$ & $63(38,2)$ & $77(46,7)$ & $17(10,3)$ & $7(4.2)$ & $1(0,6)$ & $165(100)$ \\
\hline Torna meu trabalho mais prazeroso & $79(47,9)$ & $68(41,2)$ & $12(7,3)$ & $6(3,6)$ & $0(0)$ & $165(100)$ \\
\hline
\end{tabular}






Figura 1. Pontuações das habilidades em pesquisa relatadas por 165 enfermeiros clínicos do Hospital Israelita Albert Einstein com a utilização da Escala de Autoeficácia em Pesquisa de Enfermagem (NURSES adaptada). São Paulo, Brasil. Setembro de 2012

Entretanto, poucos enfermeiros eram capazes de redigir um artigo nesses idiomas (23,6 e 7,9\%, respectivamente). Apenas 2,4\% eram capazes de ler e compreender francês e nenhum era capaz de redigir naquele idioma.

Capacidades ainda menores para redigir estavam significativamente associadas ao maior tempo decorrido desde a graduação $(\mathrm{p}=0,008)$. Como esperado, a melhor capacidade de redação estava associada aos pós-graduados $(\mathrm{p}=0,022)$, sendo que o título de doutorado estava associado a uma melhor capacidade geral $(p=0,044)$ e a uma melhor capacidade para pesquisa $(p=0,018)$. A docência também favoreceu positivamente a capacidade geral para pesquisa desses enfermeiros $(p=0,011)$, assim como a capacidade de buscar informações e utilizar as habilidades de práticas baseadas em evidência e de uso de conhecimentos teóricos $(\mathrm{p}=0,004$; 0,031 , e 0,002 , respectivamente). A instituição acadêmica de origem (pública ou privada), a participação em cursos de treinamento em pesquisa e apresentação dos resultados de suas pesquisas em publicações ou eventos não foram estatisticamente significativos em relação às habilidades de pesquisa.

Os enfermeiros com título de mestrado tinham crenças mais positivas sobre os benefícios da pesquisa e melhores habilidades para pesquisa (Tabela 2). A participação em grupos de pesquisa foi o fator de maior influência nesse aspecto (Tabela 3). Do mesmo modo, ter apresentado resultados de pesquisa em eventos científicos influenciou positivamente as crenças dos enfermeiros em relação à pesquisa $(\mathrm{p}=0,001)$, independentemente de serem investigador principal $(p=0,002)$ ou coautores
Tabela 2. Associação entre crenças/habilidades e título de mestrado

\begin{tabular}{lcccc}
\hline Crenças e habilidades & $\begin{array}{c}\text { Título de } \\
\text { mestrado }\end{array}$ & $\begin{array}{c}\text { Sem título } \\
\text { de mestrado }\end{array}$ & $\begin{array}{c}\text { Valor } \\
\text { de p }\end{array}$ \\
\cline { 2 - 3 } Mediana & 10 & & 8,4 & $<0,001$ \\
\hline Crenças (0-10) & 7,4 & 6,5 & 0,011 \\
Habilidades gerais (0-10) & 3,3 & & 3,3 & 0,001 \\
Idiomas (0-10) & 7,5 & 6,9 & 0,018 \\
Revisão de literatura (0-10) & 6,7 & & 5,8 & 0,121 \\
Compreensão da literatura de pesquisa e & & & \\
avaliação da qualidade dos estudos (0-10) & 7,5 & 7,5 & 0,033 \\
Uso de teoria (0-10) & 7,5 & 7,5 & 0,051 \\
Compreensão de evidências (0-10) & & & \\
\hline
\end{tabular}

Tabela 3. Associação entre crenças/habilidades e participação de enfermeiros clínicos no grupo de pesquisa acadêmica

\begin{tabular}{lccccc}
\hline & Sim & & Não & & Valor \\
\cline { 2 - 2 } & Mediana & & Mediana & de p \\
\hline Crenças (0-10) & 10 & & 8,4 & $<0,001$ \\
Habilidades gerais (0-10) & 7,1 & & 6,6 & 0,049 \\
Idiomas (0-10) & 3,3 & & 3,3 & 0,062 \\
Revisão da literatura (0-10) & 7,5 & & 6,9 & 0,017 \\
Compreensão da literatura de pesquisa & 0 & 6,3 & & 5,8 & 0,679 \\
avaliação da qualidade dos estudos (0-10) & & & & \\
Uso de teoria (0-10) & 8,3 & & 7,5 & 0,006 \\
Compreensão de evidências (0-10) & 10 & & 7,5 & $<0,001$ \\
Redação científica (0-10) & 7,1 & & 7,5 & 0,488 \\
\hline
\end{tabular}

$(\mathrm{p}=0,032)$. Um total de $77,5 \%$ dos respondentes esperavam realizar alguma pesquisa no futuro e $37,5 \%$ demonstraram interesse em processos de inovação.

\section{DISCUSSÃO}

Neste estudo, estabelecemos um perfil detalhado da experiência em pesquisa, crenças e autoeficácia na pesquisa de enfermeiros clínicos no primeiro hospital admitido na Jornada Magnet ${ }^{\circledR}$ na América do Sul.

Graus variáveis de experiência em pesquisa foram relatados pelos 165 respondentes. De modo geral, os enfermeiros clínicos foram positivos em suas crenças, assim como na percepção de suas próprias habilidades de pesquisa, que foram mais positivas do que as relatadas na literatura ${ }^{(6,7)}$. Observou-se que os enfermeiros que fizeram cursos de especialização tinham uma maior percepção das dificuldades associadas à redação científica. Talvez decorrentes de uma visão mais crítica de suas próprias limitações por terem sido expostos a essa experiência. Por outro lado, algumas barreiras, como tempo insuficiente para realizar pesquisa, podem ter prejudicado a capacidade desses profissionais para fa- 
zer e publicar pesquisas ${ }^{(8,9)}$. Além disso, como a participação em publicações reforçou essas crenças positivas, mas não a percepção de suas próprias habilidades de pesquisa, esses resultados indicam que os enfermeiros clínicos podem não ter sido os investigadores principais dos estudos publicados.

Outro aspecto que pode ter contribuído para a baixa produção científica é a dificuldade em analisar criticamente textos e artigos científicos escritos em outros idiomas. Na realidade, a capacidade de análise crítica de textos científicos escritos em inglês foi pouco avaliada pelos respondentes. Inglês é o idioma internacional da ciência ${ }^{(10)}$, mas, para os autores cuja língua materna não é o inglês, a redação de manuscritos científicos representa um grande desafio.

Já foi sugerido que os enfermeiros clínicos são altamente motivados para participar de pesquisas, mas que não têm confiança para iniciar e implementar projetos de pesquisa ${ }^{(11)}$. Com poucas exceções, a pesquisa em enfermagem não tem sido uma parte característica das atividades da enfermagem clínica. É raro ter uma equipe com habilidades e formação acadêmica necessárias para facilitar as pesquisas ${ }^{(7)}$. Muitos enfermeiros clínicos sentem-se intimidados pela pesquisa. As pesquisas realizadas anteriormente estão em conformidade com este estudo, tendo sido relatado que os enfermeiros clínicos têm "medo do desconhecido", "falta de capacitação ou confiança para realizar pesquisas", ou "a crença de que não possuem habilidades para realizar pesquisas, o que impede a realização de projetos de pesquisa"(12).

Neste estudo, a maioria dos enfermeiros percebeu que as seguintes habilidades eram bem desenvolvidas: a capacidade de procurar informações de maneira eficaz, habilidades teóricas, prática baseada em evidência, capacidade de redação e leitura, e a compreensão de inglês e espanhol. A conclusão de mestrado ou doutorado, assim como a participação em grupos de pesquisa e docência, também teve influência positiva na percepção dos enfermeiros sobre suas habilidades. A autopercepção dos enfermeiros clínicos sobre as habilidades de pesquisa foi, na verdade, maior do que relatada anteriormente ${ }^{(6,7)}$. Entretanto, nossos resultados indicaram que essas habilidades pareciam favorecer mais a prática baseada em evidência do que a pesquisa em enfermagem. A baixa produção científica relatada aqui reforça essa ideia, em conformidade com a noção de que apenas enfermeiros isolados realizam pesquisas e de que as publicações são raras $^{(13)}$.

Nossos achados também indicaram que o principal impacto da pesquisa em enfermagem percebido pelos respondentes está associado à melhora da imagem institucional. Esse valor é fortemente reconhecido pelos funcionários dessa instituição. Isso pode ser explicado pelo fato de que o hospital é reconhecido pela excelência na qualidade de cuidado aos pacientes, sendo uma instituição do mais alto nível no Brasil. Em 2012, o HIAE foi reconhecido com a premiação SciVal Brazil (Elsevier), que consagra as instituições brasileiras de pesquisa com excelência notável na produção científica. O HIAE recebeu o prêmio na categoria "Citações por documento". Nossos achados contrastaram com os estudos anteriores ${ }^{(5)}$, indicando que as respostas mais positivas dos enfermeiros clínicos sobre suas visões do impacto da pesquisa estão associadas aos padrões de cuidados com os pacientes e o desenvolvimento de habilidades específicas.

A condução e a aplicação de pesquisas não têm sido uma prioridade para os enfermeiros em geral, apesar da crença amplamente aceita de que a pesquisa em enfermagem é relevante. Enquanto a maior parte dos enfermeiros clínicos busca a prática baseada em evidência, são aparentemente relutantes e apáticos quanto à condução de pesquisa em enfermagem ${ }^{(13)}$. Na realidade, os enfermeiros de instituições de saúde acadêmicas e não acadêmicas mencionaram pouco a médio interesse em pesquisa, sugerindo que este seja um aspecto presente universalmente ${ }^{(4)}$. Nossos resultados apoiam essa noção, como pode ser evidenciado pelo fato de que o nível espontâneo de adesão dos enfermeiros clínicos para responder os questionários foi baixo. Para a maioria dos enfermeiros, o tema da pesquisa parecia muito distante e despertava pouco interesse. A recente contratação de um enfermeiro pesquisador, a falta de familiaridade com um novo departamento - o Núcleo de Pesquisa em Enfermagem e Multiprofissional -, que não existia na instituição, também podem ter contribuído para isso. A observação de que a pesquisa em enfermagem não é uma prioridade para os enfermeiros clínicos é incompreensível, uma vez que os enfermeiros que trabalham em hospitais são bem posicionados para identificar problemas da prática clínica encontrados diariamente à beira do leito e podem ajudar a construir evidências para suas práticas ${ }^{(12)}$. Observou-se pouca participação nos grupos de pesquisa e, consequentemente, pouca experiência em pesquisa. Isso é consistente com análises realizadas anteriormente, mostrando que os enfermeiros desejam firmar parcerias com escolas de enfermagem para fazer pesquisas $^{(14)}$. Porém, há vários desafios, como dificuldade em negociar propostas de pesquisa de interesse comum ao corpo docente da universidade e aos enfermeiros clínicos. Alguns enfermeiros preferem liderar suas próprias pesquisas a firmar parceria com faculdades de enfermagem ${ }^{(15)}$. Particularmente, os enfermeiros clínicos relatam que desejam conduzir estudos que se traduzam em benefícios para a vida real. 
Em geral, nosso diagnóstico do perfil acadêmico e científico dos enfermeiros clínicos registrados no HIAE indicou alta heterogeneidade de perfis, assim como algumas idiossincrasias e crenças nessa população, que deveriam ser consideradas na implantação de um programa destinado ao desenvolvimento das práticas de pesquisa em enfermagem. Entretanto, uma limitação desse estudo foi o fato de que os respondentes podem não representar o perfil dos enfermeiros clínicos da instituição. Os participantes que optaram por completar a pesquisa podem ter tido uma maior percepção da relevância e do valor da pesquisa em enfermagem para a prática da atividade.

Ainda há vários desafios ao desenvolvimento de melhores estruturas institucionais para apoiar e integrar a pesquisa no ambiente clínico, e mostrar que os achados de pesquisa são relevantes para os clínicos. Os modelos mais comuns incluem a presença de um conselho de enfermagem do hospital orientado por pesquisadores acadêmicos; um enfermeiro pesquisador com titulação acadêmica contratado; um enfermeiro pesquisador em período parcial; e um enfermeiro pesquisador em período integral ${ }^{(16)}$. O último modelo é adotado em nossa instituição. O Núcleo de Pesquisa e Enfermagem e Multiprofissional foi criado para promover e apoiar as práticas de pesquisa e o treinamento entre os enfermeiros clínicos e a equipe multidisciplinar do hospital. No HIAE, o enfermeiro pesquisador tem várias funções: pesquisador independente, docente/orientador, consultor e mentor, como relatado de maneira semelhante em outras instituições no mundo todo ${ }^{(17)}$.

O diagnóstico do perfil acadêmico dos enfermeiros identificados aqui indica que são necessários diferentes tipos de treinamento. Portanto, propomos o seguinte programa de treinamento: nível básico, com cursos de metodologia científica ( 8 horas), pesquisa bibliográfica (programas regulares da biblioteca) e redação científica (16 horas). Para os níveis de experiência intermediário e avançado, recomendamos a análise crítica dos artigos e a redação científica (40 horas). Para formação contínua, propomos a implantação de reuniões mensais. Além disso, nosso programa oferece um prêmio de pesquisa para as contribuições científicas em enfermagem clínica e implementação de estratégias para maior visibilidade. Tais contribuições científicas são avaliadas por meio de indicadores quantificáveis (artigos publicados, citações, propostas de inovações, patentes e projetos colaborativos).

$\mathrm{O}$ aprimoramento das habilidades de pesquisa dos enfermeiros clínicos é discutido no mundo todo. Embora esse estudo tenha sido conduzido em um hospital privado no Brasil, nossos achados podem ser úteis para outras organizações com perfis semelhantes em diferentes regiões e países. De maneira semelhante, nosso programa proposto com base em dados também pode contribuir para discussões futuras sobre a implantação da pesquisa em enfermagem em instituições não acadêmicas, especialmente aquelas que buscam obter a designação Magnet ${ }^{\circledast}$. A compreensão da experiência em pesquisa, as habilidades e as crenças da equipe de enfermagem clínica de uma instituição podem ajudar na implantação de projetos de pesquisa, assim como na escolha das melhores estratégias de treinamento. Essa análise é sempre necessária para reduzir o risco de importar ações inapropriadas de outros estudos, o que pode não corresponder à realidade institucional. Não há um modelo ideal descrito na literatura para aumentar o envolvimento dos enfermeiros clínicos na pesquisa em enfermagem. Entretanto, é importante refletir sobre as maneiras que levam ao desenvolvimento eficaz dos enfermeiros clínicos na pesquisa em enfermagem e considerar o perfil de enfermeiros que realizam pesquisa à beira do leito. Nem todo enfermeiros clínicos têm o desejo e a aptidão para essa tarefa. Além disso, até o momento, não há um estudo disponível que possibilite a identificação da proporção ideal de enfermeiros pesquisadores para a equipe de enfermeiros clínicos. No futuro, pode-se reconhecer a necessidade de contratar mais enfermeiros pesquisadores, não apenas para suporte ou orientação, mas também para desenvolver pesquisa de alta qualidade. Essa será uma transformação real na produção de conhecimento científico pelos enfermeiros hospitalares e possibilitará a avaliação e os acompanhamentos adequados da vocação de cada enfermeiro.

Nossos achados devem contribuir para a elaboração de uma proposta de programa de pesquisa adequada ao nível de experiência acadêmica dessa população.

\section{CONCLUSÕES}

Neste estudo, estabelecemos um perfil detalhado da experiência em pesquisa, crenças e autoeficácia na pesquisa de enfermeiros clínicos no primeiro hospital da América do Sul a ser admitido na Jornada Magnet ${ }^{\circledR}$. Os enfermeiros clínicos relataram graus variáveis de experiência em pesquisa. Foram observadas crenças e percepções positivas sobre as habilidades de pesquisa. Contudo, a percepção positiva não se traduziu em efetiva produção científica.

\section{REFERÊNCIAS}

1. Dyniewicz AM, Rivero de Gutiérrez MG. [Research methodology for nurses at a university hospital]. Rev Latam Enferm. 2005;13(3):354-63. 
2. Drenkard K, Wolf G, Morgan SH. Editors Magnet: The next generation Nurses making the difference. Maryland: American Nursing Credentialing Center; 2011.

3. Deave T. Research nurse or nurse researcher: how much value is placed on research undertaken by nurses? J Res Nurs. 2005;10(6):649-57.

4. Silka CR, Stombaugh HA, Horton J, Daniels R. Nursing research in a nonacademic health system: measuring knowledge, attitudes, and behaviors. J Nurs Adm. 2012;42(7-8):386-92.

5. Appleton L, Smith K, Wyatt D. Nursing involvement in a practice development and research unit. Cancer Nurs Practice. 2010;9(7):18-22.

6. Sweson-Britt E, Reineck C. Research education for clinical nurses: a pilot study to determine research self-efficacy in critical care nurses. J Cont Educ Nurs. 2009;40(10):454-61

7. Jamerson PA, Vermeersch P. The role of the nurse research facilitator in building research capacity in the clinical setting. J Nurs Adm. 2012;42(1):21-7.

8. Atkinson M, Cashy J, Turkel M. Overcoming Barriers to Research in a Magnet Community Hospital. J Nurs Care Qual. 2008;23(4):362-8.

9. Turkel $M$, Ferket $K$, Reidinger G, Beatty DE. Building a nursing research fellowship in a community hospital. Nurs Economics. 2008;26(1):26-34.
10. English as the international language of science. Res Trends. [Internet]. 2008;(6) [cited 2013 Apr 12]. Disponível em: http://http://www.researchtrends.com/ issue6-july-2008/english-as-the-international-language-of-science/

11. Willson P, Madary A, Brown J, Gomez L, Martin J, Molina T. Using the forces of magnetism to bridge nursing research and practice. J Nurs Adm. 2004; 34(9):393-4.

12. Kelly KP, Turner A, Gabel Speroni KG, McLaughlin MK, Guzzetta CE. National survey of hospital nursing research, part 2: facilitators and hindrances. J Nurs Adm. 2013;43(1):18-23.

13. Wilson C, Sylvanus T. Generating enthusiasm for nursing research. JNurs Adm. 2005;35(5):220-3.

14. Dols JD, Bullard K, Gembol L. Setting a nursing research agenda. J Nurs Adm. 2010;40(5):201-4.

15. Latimer R, Kimbell J. Nursing research fellowship: building nursing research infrastructure in a hospital. J Nurs Adm. 2010;40(2):92-8.

16. Wilson B, Kelly L, Reifsnider E, Pipe T, Brumfield V. Creative approaches to increasing hospital-based nursing research. J Nurs Adm. 2013;43(2):80-8.

17. Albert N, Siedlecki SL. Developing and implementing a nursing research team in a clinical setting. J Nurs Adm. 2008;38(2):90-6. 